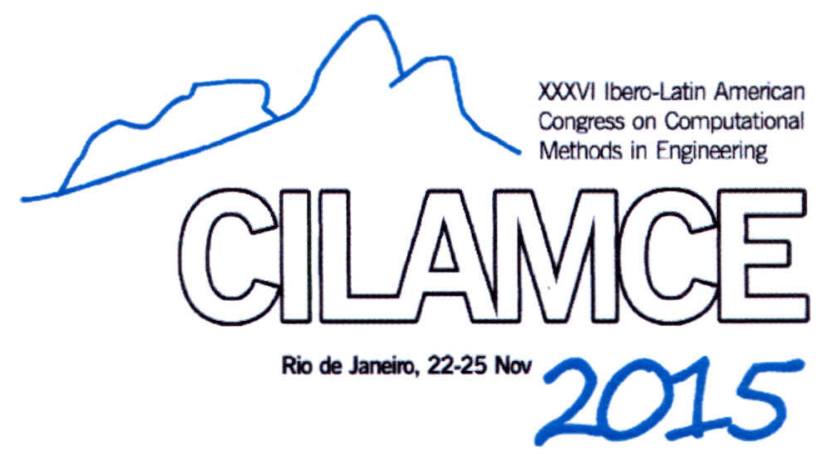

\title{
3D FINITE ELEMENT MODEL FOR MECHANICAL AND CHEMO- MECHANICAL DEFORMATION IN SEDIMENTARY BASINS
}

\author{
André Brüch \\ Samir Maghous \\ andrebruch@hotmail.com \\ samir.maghous@ufrgs.br \\ Federal University of Rio Grande do Sul, Department of Civil Engineering \\ Av. Osvaldo Aranha 99, $3^{\text {rd }}$ floor, Centro - CEP 90035-190 - Porto Alegre, RS, Brazil
}

\begin{abstract}
Sedimentary basins result from deposition of an appreciable amount of sediments which are transformed into rock through natural phenomena involving mechanical and chemo-mechanical deformation. Purely mechanical deformation prevails in the upper layers of basins, whereas chemo-mechanical deformation dominates at depth, where effective stresses and temperature are higher. The aim of the present contribution is to provide a comprehensive $3 D$ framework for modeling both mechanical and chemo-mechanical compaction in sedimentary basins. Extending concepts previously proposed for the modeling of purely mechanical compaction in finite poroplasticity, chemo-mechanical deformation induced by Intergranular Pressure-Solution (IPS) is addressed by means of additional viscoplastic terms in the state equations of the porous material. Special attention is given on the analysis of the effects of large irreversible porosity changes on the poromechanical properties of the sediment material. The sedimentary basin is modeled as a fully saturated poro-elasto-visco-plastic material undergoing large strains. The numerical simulation is performed through the finite element method. An oceanic sedimentary basin is modelled as a horizontal infinite layer where sediments deposition occurs over tens of millions of years. The evolution on time of the porous material constitutive model is presented and analyzed in conjunction with the overall behavior of the sedimentary basin.
\end{abstract}

Keywords: Sedimentary Basin, Poroviscoplasticity, Chemo-Mechanical Compaction, Intergranular Pressure-Solution. 


\section{INTRODUCTION}

Sedimentary basins result from the deposition of an appreciable amount of sediments which are transformed into rock through a natural phenomenon called diagenesis. Reconstructing the stress and deformation history of a sedimentary basin is an important and challenging problem in geoscience to better understand the geological history of the planet. It is also of great interest to the oil industry, since formation of natural hydrocarbons occurs almost entirely within sedimentary rocks. However, accurate prediction of rock porosity through time is a hard task and a key challenge still remains to the constitutive model of the porous material due to the coupled thermo-hydro-chemo-mechanical nature of the problem.

The compaction process of a sedimentary basin in fully saturated conditions can be divided into two main groups: purely mechanical and chemo-mechanical. The process of mechanical compaction takes place through water expulsion from the porous material, thus resulting in grain repacking and volume reduction, involving grain reorientation, sliding, deformation, and breakage. After this initial porosity loss, further porosity reduction is accomplished by the process of chemo-mechanical compaction resulting from a waterassisted physico-chemical process known as Intergranular Pressure-Solution (IPS) (Schneider et al., 1994). Such a compaction process is typically assumed to viscous and it is usually referred to as viscous compaction. Its rheological constitutive relation is often written as a relationship between effective stress and strain rate (Yang, 2000).

For a chemically closed system at grain scale, Intergranular Pressure-Solution process is traditionally described by a three steps mechanism: (1) dissolution at intergranular interfaces, (2) diffusion of solutes inside the contact between two grains, and (3) precipitation on the surface of the grains in contact with the pore fluid. The slowest of the three serial processes controls the overall deformation. The rate-controlling process may even change during the compaction process (Gundersen et al., 2002) as the whole process depend on many factors as effective stress, pressure, temperature, cementation level, grain size, grain mineralogy, solution chemistry and burial rate (Tada and Siever, 1989).

Two models of grain contact structures have been proposed, namely the 'water film diffusion' and 'island \& channel' model. In the thin film model, the grain contacts are assumed to consist of a thin water film trapped inside contacts and cannot be squeezed out by applied stress (Weyl, 1959; Rutter, 1976). The 'island \& channel' model assumes that the interface possesses dynamically roughening contacts of islands and channels penetrated by water (Ghoussoub and Leroy, 2001). In both models, water in between the grain contacts is important to dissolve solid minerals and to transport material out of the contact areas. In natural conditions, pressure solution may only occur where water is present, and inhibited where dry or other fluids such as hydrocarbons filled (Zhang and Spiers, 2005). While controversy persists in the literature as to which is the most appropriate grain boundary model (Hickman and Evans, 1991; Schutjens and Spiers, 1999), their mathematical treatment is equivalent so that no distinction is needed for present purposes (de Meer and Spiers, 1997).

Purely mechanical deformation prevails in the upper layers of the basins, whereas chemomechanical deformation dominates at depth, where effective stresses and temperature are higher (Schmidt and McDonald, 1979). However, there exist a transition zone where mechanical and chemo-mechanical deformation may behave as strongly interdependent processes (Hu and Hueckel, 2007). The location of the transition zone ranges from a depth of a few hundred meters to more than a kilometre depending on the nature of the porous material and field conditions (Schneider et al., 1996). 
The aim of the present contribution is to provide a comprehensive 3D framework for modeling both mechanical and chemo-mechanical compaction in sedimentary basins. Extending concepts previously proposed for the modeling of purely mechanical compaction in finite poroplasticity, deformation by IPS is addressed by means of additional viscoplastic terms in the state equations of the porous material. Special attention is given on the analysis of the effects of large irreversible porosity changes on the poromechanical properties of the sediment material.

The sedimentary basin is modeled as a fully saturated poro-elasto-visco-plastic material undergoing large strains. Hydro-chemo-mechanical and elasticity-plasticity couplings are considered. The numerical simulation is performed through the finite element method. An oceanic sedimentary basin is modelled as a horizontal infinite layer where sediments deposition occurs over tens of millions of years. The basin mesh is made up by a single column of elements. The evolution on time of the porous material constitutive model is presented and analyzed in conjunction with the overall behavior of the sedimentary basin.

\section{POROMECHANICAL CONSTITUTIVE MODEL}

At the macroscopic scale, a porous medium can be viewed as the superposition of a solid continuum related to the deformable skeleton and a fluid phase occupying the porous space. In order to define the poromechanical problem under isothermal and quasi-static conditions, two governing equations referring to momentum and mass balance must be specified. By considering the balance of linear momentum, the equilibrium equation for the porous continuum is:

$$
\operatorname{div} \underline{\underline{\sigma}}+\rho \underline{g}=\underline{0}
$$

where $\underline{\underline{\sigma}}$ is the Cauchy total stress tensor, $\underline{g}$ is the acceleration of gravity and $\rho=\varphi \rho^{f}+(1-\varphi) \rho^{s}$ is the density of the porous material, which can be computed from the Eulerian porosity $\varphi$ (i.e. the pore volume fraction in the current configuration), the fluid density $\rho^{f}$ and the solid grains density $\rho^{s}$.

Denoting by $\phi=J \varphi$ the Lagrangian porosity, where $J$ is the Jacobian of the geometrical transformation of the skeleton particle, the second field equation refers to the fluid mass balance:

$$
\frac{d}{d t}\left(\rho^{f} \phi\right)+J \operatorname{div}\left(\rho^{f} \underline{q}\right)=0
$$

where $q$ is the filtration vector defined according to the Darcy's law:

$$
\underline{q}=\underline{\underline{k}} \cdot\left(-\underline{\nabla} p+\rho^{f} \underline{g}\right)
$$

where $p$ is the pore-pressure and $\underline{\underline{k}}$ is the permeability tensor, which is reduced to $\underline{\underline{k}}=k \underline{\underline{1}}$ when isotropy is taken into account. 
In the framework of finite poro-visco-plasticity, the constitutive behavior comprises two state equations in rate-type formulation. The first equation relates the stress rate $\underline{\underline{\dot{\sigma}}}$ and pore pressure rate $\dot{p}$ to the strain rate tensor $\underline{\underline{d}}$ :

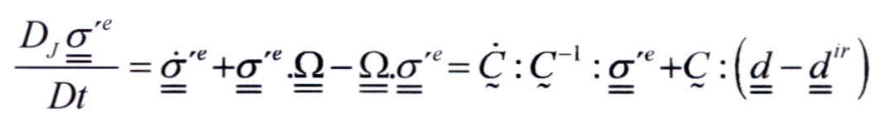

where $\underline{\underline{d}}^{i r}=\underline{\underline{d}}^{p}+\underline{\underline{d}}^{v p}$ stands for the irreversible strain rate, $\underline{\underline{d}}^{p}$ (resp. $\underline{\underline{d}}^{v p}$ ) is the plastic (resp. viscoplastic) strain rate, while $\underline{\underline{\Omega}}$ is the rotation (spin) rate tensor which aims at taking the large rotation of the elementary volume into account. This equation involves a rotational time derivative $D_{J} / D t$ of the Biot effective stress tensor $\underline{\underline{\sigma}}^{\prime e}=\underline{\underline{\sigma}}+b p \underline{\underline{1}}$, where $b$ is the Biot coefficient. It also includes a term related to the particulate derivative $\underset{\sim}{\dot{C}}$ of the tensor of drained elastic moduli $\underset{\sim}{C}$, which aims at capturing the evolution of the elastic properties with the microstructural changes due to large irreversible strains, of what is called elasticityplasticity coupling (Dormieux and Maghous, 2000).

The second state equation relates the pore volume change rate to the rate $\dot{p}$ of the pore pressure and to the strain rate $\underline{\underline{d}}$ :

$$
\dot{p}=M\left(\frac{\dot{\phi}-\dot{\phi}^{i r}}{J^{i r}}-\dot{b} t r\left(\underline{\sim}^{-1}:{\underline{\underline{\sigma^{\prime}}}}^{e}\right)-b \operatorname{tr}\left(\underline{\underline{d}}-\underline{\underline{d}}^{i r}\right)\right)+\frac{\dot{M}}{M} p
$$

where $M$ is the Biot modulus, $\phi^{i r}$ is the irreversible part of the Lagrangian porosity variation and $J^{i r}=J^{p} J^{v p}$ is the irreversible part of the Jacobian of transformation. $J^{p}$ and $J^{v p}$ are, respectively, the plastic and viscoplastic parts of the Jacobian of transformation. The terms involving $\dot{M}$ and $\dot{b}$ are related to the influence of large irreversible strains on the poroelastic properties.

The solid phase constituting the skeleton is assumed to be plastically incompressible. This implies that the volume of solid particles remains unchanged during the irreversible transformation of the porous material. In the framework of infinitesimal elastic strains, it is possible to assign the following relation, where $J \approx J^{i r}$ was taken into account:

$$
\varphi=1-\frac{1-\phi_{0}}{J^{i r}}
$$

In the present constitutive model, plasticity is used to model purely mechanical deformation while viscoplasticity represents the chemo-mechanical deformation induced by Intergranular Pressure-Solution. The plastic behavior of the porous material is based on the modified Cam-Clay model (Wood, 1990). The yield surface is given by:

$$
f^{p}\left(\underline{\underline{\sigma^{\prime}}}, p_{c}\right)=\frac{3}{2} \underline{\underline{s}}: \underline{\underline{s}}+M_{c s}^{2} p^{\prime}\left(p^{\prime}+p_{c}\right)
$$


where $p_{c}$ is the consolidation pressure and represents the hardening parameter of the model. The constant $M_{c s}$ is the slope of the critical state line. The plastic criterion depends on the Terzaghi effective stress $\underline{\underline{\sigma^{\prime}}}=\underline{\underline{\sigma}}+p \underline{\underline{1}}$. The deviatoric stress tensor $\underline{\underline{s}}$ and the mean effective stress $p^{\prime}$ are given:

$$
\underline{\underline{s}}=\underline{\underline{\sigma}}-\frac{1}{3} \operatorname{tr}(\underline{\underline{\sigma}}) \underline{\underline{1}} \quad p^{\prime}=\frac{1}{3} \operatorname{tr}\left({\underline{\underline{\sigma^{\prime}}}}^{\prime}\right)
$$

The associated plastic flow rule reads:

$$
\underline{\underline{d}}^{p}=\dot{\chi} \frac{\partial f^{p}}{\partial \underline{\underline{\sigma}}^{\prime}}, \text { with } \dot{\chi} \geq 0
$$

The assumption of plastic incompressibility of the solid phase also implies $J^{p}=1+\phi^{p}$, where $\phi^{p}$ is the plastic part of the Lagrangian porosity variation. Taking the time derivative of this relation in conjunction with the identity $\dot{J}^{p}=J^{p} t r \underline{\underline{d}}^{p}$ it is possible to obtain the flow rule for the rate of plastic porosity:

$$
\dot{\phi}^{p}=J^{p} \operatorname{tr} \underline{\underline{d}}^{p}
$$

The hardening law is object of special concern. Based on micromechanical reasoning, a specific formulation has been developed for the evolution of $p_{c}$ by determining the limit load of a hollow sphere subjected to large isotropic compression (Barthélémy et al., 2003). The advantage of this hardening law with respect to classical ones lies in the fact that it avoids the development of negative porosities under high isotropic compression (Deudé et al., 2004).

$$
p_{c}\left(J^{i r}\right)=\frac{p_{c 0}}{\ln \left(\phi_{0}\right)} \ln \left(1-\frac{1-\phi_{0}}{J^{i r}}\right)
$$

The evolution of the hardening parameter of the plastic model is associated with both plastic and viscoplastic deformation through $J^{i r}$ as they may be interdependent processes and both phenomena contribute to porosity closure. More precisely, the consolidation pressure predicted by (11) tends towards infinity when the pore space vanishes (i.e. $J^{i r} \approx J \rightarrow 1-\phi_{0}$ ).

The viscoplastic component of the strain rate is formulated adopting the following associated viscoplasticity model, where a static criterion $p_{v p}^{s}$ was defined to represent the viscoplastic yield surface of the porous material in conjunction with a dynamic surface $f_{d}^{v p}$ defined to describe its current effective stress state (Perzyna, 1966).

$$
\underline{\underline{d}}^{v p}=\frac{1}{\eta}\left\langle\frac{p_{v p}^{d}}{p_{v p}^{s}}-1\right\rangle^{n} \frac{\partial f_{d}^{v p}}{\partial \underline{\underline{\sigma}}^{\prime}}
$$

where $\langle\cdot\rangle$ is the Macaulay brackets, $\eta$ is the viscosity coefficient which incorporates the different fundamental parameters governing the creep law for pressure-solution, $n$ is the 
viscosity exponent and $p_{v p}^{d}$ is the dynamic parameter associated to the dynamic viscoplastic surface $f_{d}^{v p}\left(\underline{\underline{\sigma^{\prime}}}, p_{v p}^{d}\right)$ defined by the Cam-Clay model (same as (7) with $p_{v p}^{d}$ instead of $p_{c}$ ):

$$
p_{v p}^{d}=-\frac{3}{2} \frac{\underline{s}: \underline{\underline{s}}}{M_{c s}^{2} p^{\prime}}-p^{\prime}
$$

The evolution law for $p_{v p}^{s}$ is taken from the hardening law of $p_{c}$ on which an additional exponent $m$ is used to control the evolution rate of the viscoplastic yield surface:

$$
p_{v p}^{s}\left(J^{i r}\right)=p_{v p 0}^{s}\left(\frac{\ln \left(1-\frac{1-\phi_{0}}{J^{i r}}\right)}{\ln \left(\phi_{0}\right)}\right)^{m}
$$

Using plastic and viscoplastic hardening laws depending on both plastic and viscoplastic deformation lead to an additional non-linearity in the constitutive model when both mechanical and chemo-mechanical phenomena occur, as in the transition zone of a sedimentary basin.

Since large irreversible deformations lead to significant changes in porosity, the evolution of elastic stiffness shall be considered. The idea is to resort to micromechanical estimates to capture the influence of the plastic and viscoplastic strains on the poromechanical properties of the material. For sake of simplicity, the anisotropy induced during the loading process is disregarded. Accordingly, the bulk $K$ and shear $\mu$ moduli of the porous medium now appear as functions of the Eulerian porosity $\varphi$ as well as of the elastic properties of the solid phase $k^{s}$ and $\mu^{s}$. More precisely, we adopt herein the Hashin-Shtrikman upper bounds which are known to reasonably model the elastic properties of isotropic porous media (Hashin, 1983):

$$
K(\varphi)=\frac{4 k^{s} \mu^{s}(1-\varphi)}{3 k^{s} \varphi+4 \mu^{s}} \quad \mu(\varphi)=\frac{\mu^{s}(1-\varphi)\left(9 k^{s}+8 \mu^{s}\right)}{k^{s}(9+6 \varphi)+\mu^{s}(8+12 \varphi)}
$$

Biot coefficient $b$ and Biot modulus $M$ are deduced from the following equations (Coussy, 2004):

$$
b(\varphi)=1-\frac{K(\varphi)}{k^{s}} \quad M(\varphi)=\frac{k^{s}}{b(\varphi)-\varphi}
$$

The effects of microstructural changes on the evolution of the permeability coefficient of the porous medium $k$ may be modeled by means of the Kozeny-Carman formula:

$$
k(\varphi)=k_{0} \frac{\varphi^{3}\left(1-\phi_{0}\right)^{2}}{\phi_{0}^{3}(1-\varphi)^{2}}
$$




\section{FINITE ELEMENT DISCRETIZATION}

Assessment of the poromechanical state requires the determination of the temporal evolution of the geometric transformation as well as the pore-pressure changes. This shall be achieved by solving the boundary value problem defined by the set of governing equations together with the constitutive and complementary equations. The particularity of large strains is that all equations actually refer to the mechanical system in its current configuration, which is a priori unknown. The finite element procedure used for assessing the evolution of the porous medium under consideration will be briefly outlined hereafter (Bernaud et al., 2002).

The analysis is based on the implementation of the updated Lagrangian scheme (Bathe, 1996). This approach is based on the same procedures used by total Lagrangian formulations, but instead of being referred to the initial configuration, all static and kinematic variables are referred to the last calculated configuration, say at time $t$. The unknown variables are then updated in each time step $\Delta t$. The discretized form of the problem is obtained from weak formulation of the equilibrium and fluid mass balance equations at time $t+\Delta t$.

Twenty-nodes hexahedra are used for geometry discretization (Fig. 1). A piecewise quadratic polynomial function is adopted to approximate the displacement, while piecewise linear function is adopted for pore-pressure variations. The resulting system from finite element discretization is given below:

$$
\left[\begin{array}{ll}
\underline{\underline{K}}_{U U} & \underline{\underline{K}}_{U P} \\
\underline{\underline{K}}_{P U} & \underline{\underline{K}}_{P P}
\end{array}\right]\left[\begin{array}{l}
\underline{U} \\
\underline{P}
\end{array}\right]=\left[\begin{array}{l}
\underline{F}_{U}(\underline{U}, \underline{P}) \\
\underline{F}_{P}(\underline{U}, \underline{P})
\end{array}\right]
$$

where $\underline{\underline{K}}_{I J}$ are the global stiffness sub-matrices and $\underline{F}_{I}$ the global force sub-vectors. Global vectors $\underline{U}$ and $\underline{P}$ are respectively the nodal displacements and pore-pressure difference.

It is emphasized that, for a given configuration at time $t$, the system above is highly nonlinear due to the physical non-linearities (plasticity and viscoplasticity) and geometrical nonlinearities (large strains). An iterative method is adopted for solving this system by implementing an appropriate algorithm until it is satisfied up to a required tolerance.

A main specificity of the problem lies in the fact that a sedimentary basin is an open system due to the continuous accretion of material at the top of the basin during the deposition phase. This requires an appropriate technique to overcome the difficulty of dealing with an open system in the context of the finite element method. The activation/deactivation method is used to simulate the accretion process (Bernaud et al., 2006). In the framework of such method, the real open material system is simulated as a fictitious closed one.

Figure 1 illustrates the basin construction problem, where $L_{0}$ corresponds to the sea level and $H(t)$ corresponds to the top of the sediment layer. The evolution in time of the latter must correspond to that of the real system. The simulation of the accretion phase takes place by subdividing its period $T$ into $n$ subintervals $\left[t_{i-1}, t_{i}\right]$ with $t_{0}=0$ and $t_{n}=T$. During the time increment $\Delta t_{i}=t_{i}-t_{i-1}$, the sediments supply corresponds to a height of $\Delta H_{i}$ 


$$
\Delta H_{i}=\int_{t_{i-1}}^{t_{i}} \frac{\dot{M}_{d}(x, y, t)}{\rho_{0}(x, y, t)} d t
$$

where $\dot{M}_{d}$ is the rate of sediment mass accretion per unit area and $\rho_{0}$ represents the mass density of the deposited material.

Before sediment deposition, all finite element layers constituting the system behave as sea water. This is achieved by setting a hydrostatic total stress and pore-pressure state to these elements. It is also necessary to set the sea water density $\rho^{f}$ and a high permeability coefficient (when compared to the sediment material) $|k|>>k_{0}$. For a sea water element (i.e. non-activated element) located at $z=H_{i}$, stress and pore-pressure are set to:

$$
\underline{\underline{\sigma}}=-\rho^{f} g\left(L_{0}-H_{i}\right) \underline{\underline{1}} \quad p=\rho^{f} g\left(L_{0}-H_{i}\right)
$$

For every advanced subinterval $\Delta t_{i}$, the properties of a corresponding layer with thickness $\Delta H_{i}$ are changed in conjunction with the hydraulic and elastic properties of the deposited material. This process starts at the bottom of the basin $(z=0)$ and continues upwards until the accretion phase ends.

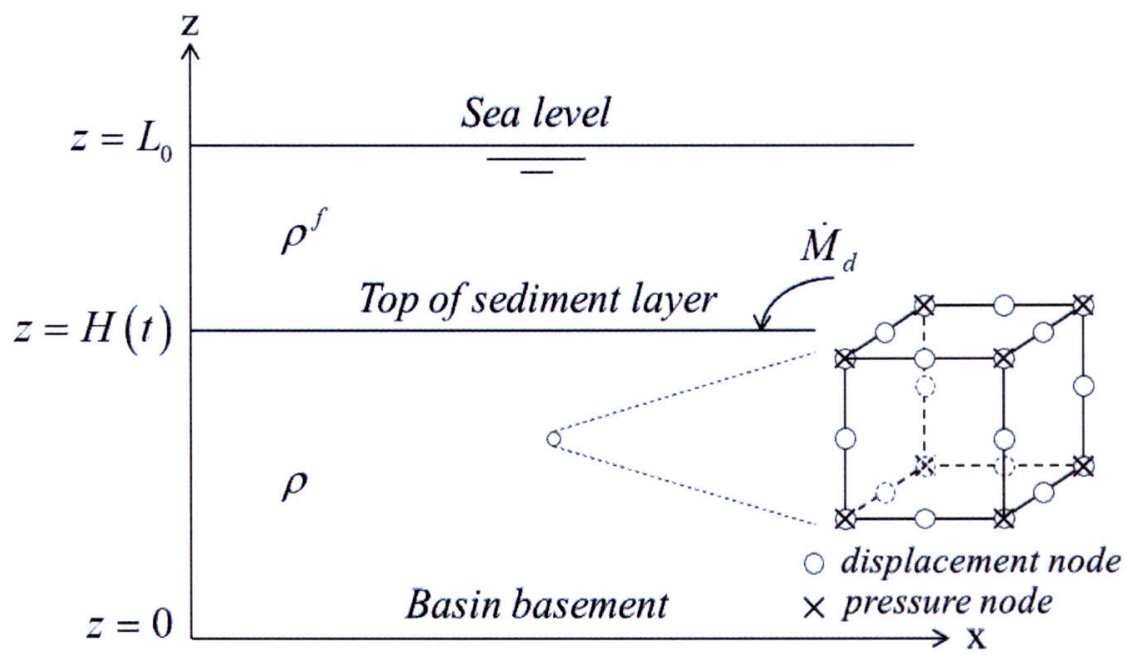

Figure 1. Schematic geometry of the basin and the finite element characteristics

\section{COMPUTATIONAL RESULTS}

The poromechanical constitutive model is used to simulate the formation and deformation processes of a sedimentary basin. No horizontal forces deriving from tectonic movements are considered, thus the whole deformation process is controlled only by gravitational forces. Therefore, a single column of elements is sufficient to simulate an oceanic basin as a horizontal infinite layer. 
The initial geometry of the model consists in a column with $6 \mathrm{~km}$ height and horizontal sides $L_{x}=L_{y}=100 \mathrm{~m}$ (actually $L_{x}$ and $L_{y}$ values do not matter). The mesh is divided into 60 equal parts in the vertical direction, resulting in cubic hexahedra elements of sides $L=100 \mathrm{~m}$. The considered situation has the following characteristics (Maghous et al., 2013): the sea bed located at $z=0$ is considered rigid and impermeable; the sea level is considered fixed at $L_{0}=8000 \mathrm{~m}$; the rate of sediment deposition is constant and equal to $100 \mathrm{~m} / \mathrm{My}$ along the accretion phase of the basin, which starts at $t=0$ and takes $T_{\text {sed }}=60$ million years, resulting in a quantity of material which would correspond to a vertical column of $6 \mathrm{~km}$ in the absence of compaction, that is if the deposited material was rigid. Figure 2 presents a sketch of the basin geometry in conjunction with the following boundary conditions:

$$
\begin{gathered}
\text { in } \quad x=0 \text { and } x=L_{x} \quad \rightarrow \quad \underline{\xi}_{-} \cdot \underline{e}_{x}=0 \text { and } \underline{q}_{\underline{e}}=0 \\
\text { in } y=0 \text { and } y=L_{y} \quad \rightarrow \quad \underline{\xi} \cdot \underline{e}_{y}=0 \text { and } \underline{q} \cdot \underline{e}_{y}=0 \\
\text { in } \quad z=0 \quad \rightarrow \quad \underline{\xi}_{\cdot} \underline{e}_{z}=0 \text { and } \underline{q} \cdot \underline{e}_{z}=0 \\
\text { in } \quad z=H(t) \rightarrow \quad \underline{T}=-\rho^{f} g\left(L_{0}-H(t)\right) \underline{e}_{z} \text { and } p=\rho^{f} g\left(L_{0}-H(t)\right)
\end{gathered}
$$

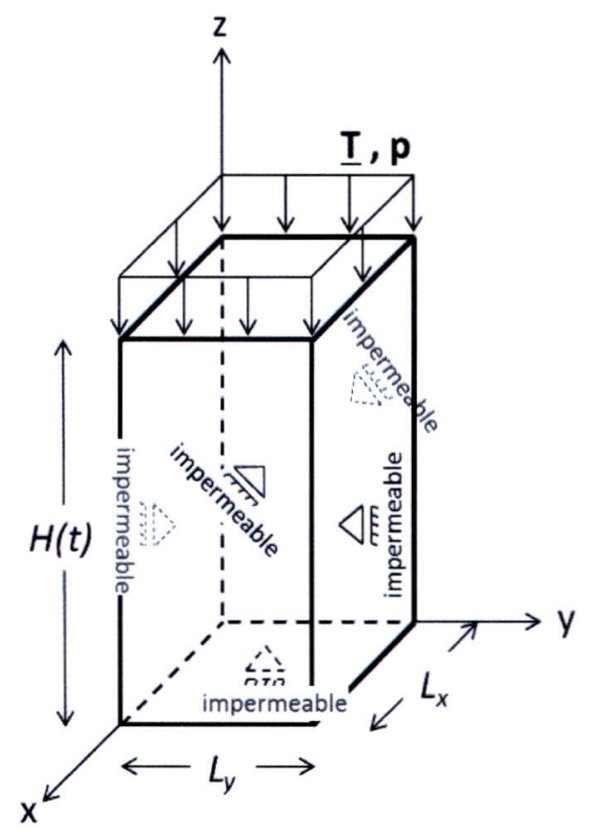

Figure 2. Basin sketch and boundary conditions

In order to facilitate the study of the evolution in time of the proposed constitutive model and its capabilities to simulate the different deformation processes of a sedimentary basin submitted to gravitational compaction, the same initial properties are considered for the sediment material along the whole accretion phase. Initial material data are: density $\rho_{0}=1.37 \times 10^{3} \mathrm{~kg} / \mathrm{m}^{3}$, porosity $\phi_{0}=0.72$, Young modulus $E_{0}=10^{3} \mathrm{MPa}$, Poisson's ratio $v_{0}=0.33$, Biot coefficient $b_{0}=0.98$, Biot modulus $M_{0}=2.22 \times 10^{5} \mathrm{MPa}$, permeability 
$k_{0}=10^{-9} \mathrm{~m}^{2} \times(\mathrm{MPa} \times s)^{-1}$, consolidation pressure $p_{c 0}=1.5 \mathrm{MPa}$, viscoplastic static criterion $p_{v p 0}^{s}=2 \mathrm{MPa}$. The viscosity coefficient is $\eta=1 \mathrm{GPa} \times M y$. The slope of the critical state line of the Cam-Clay model is the same for plasticity and viscoplasticity $M_{c s}=1.2$. The viscosity exponent of creep law is taken $n=1$ and the exponent of $p_{v p}^{s}$ evolution law is taken $m=0.5$. Sea water density is considered constant $\rho^{f}=10^{3} \mathrm{~kg} / \mathrm{m}^{3}$.

Figure 3 shows the gravitational compaction law of the basin up to $t=120 \mathrm{My}$. As it can be seen, the thickness of the basin is about $H=3500 \mathrm{~m}$ at the end of accretion phase $t=T_{\text {sed }}=60 \mathrm{My}$, which represents a compaction level of $41 \%$. This emphasizes the need to deal with a constitutive model for the sedimentary material in the context of finite strain taking into account the evolution of the poromechanical properties. The compaction rate of the basin at $t=T_{\text {sed }}=60 \mathrm{My}$ is $43 \mathrm{~m} / \mathrm{My}$. At time $t=2 T_{\text {sed }}=120 \mathrm{My}$, the basin height is $H=2730 \mathrm{~m}$ corresponding to $54 \%$ of compaction. At this moment, the compaction rate is about $3 \mathrm{~m} / \mathrm{My}$, indicating that the deformation process related to gravitational compaction is almost stabilized, as it can be observed in the compaction law curve.

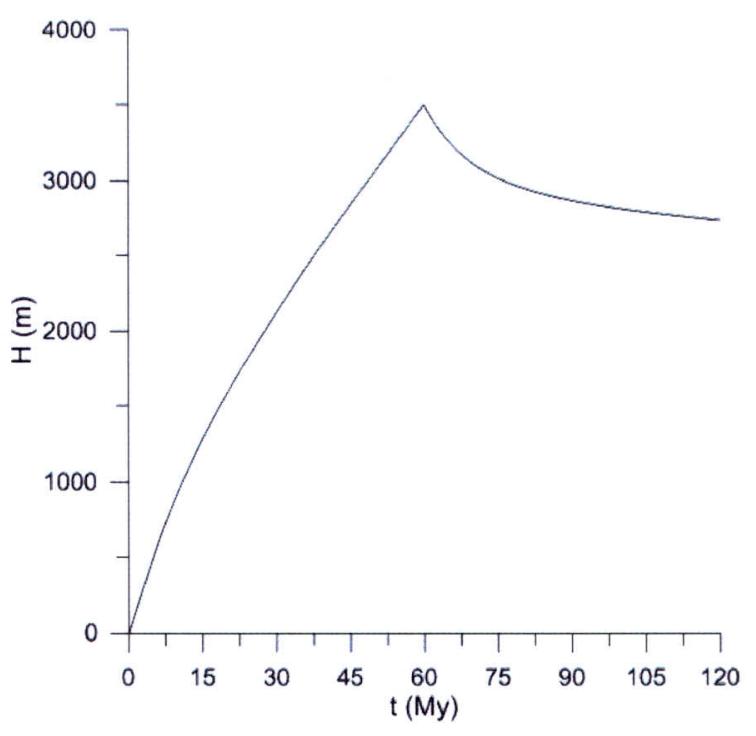

Figure 3. Compaction law

Now we focus on analyzing the evolution on time of the porous material constitutive model and the behavior of the sedimentary basin from $t=0$ and $t=120 \mathrm{My}$ by presenting some basin profiles at four different ages: $t=30,60,90$ and $120 \mathrm{My}$. Figure 4 exhibits the Jacobian of irreversible transformation $J^{i r}$ and Eulerian porosity $\varphi$ profiles along the basin thickness for the selected ages. Recalling that the porosity law of the constitutive model is function of the Jacobian of irreversible transformation, it can be readily observed that even at $t=120 \mathrm{My}$ a considerable layer $480 \mathrm{~m}$ thick remains in the elastic regime on the upper crust of the basin: while $J^{i r}=1$ (i.e. plastic and viscoplastic phenomena did not occur) porosity keeps unchanged. This means that the upper layer of the basin remained practically undeformed, as elastic deformation is considered to remain infinitesimal in the present model. 
It can also be noted that the compaction rate declines with time: since permeability is linked to porosity through Kozeny-Carman model, as porosity decreases its permeability also decreases, reducing the velocity of excess pore-pressure dissipation inside the basin. At $t=30 \mathrm{My}$ the porosity at the bottom of the basin is $30 \%$. At $t=60 \mathrm{My}$, which corresponds to the end of the accretion phase, porosity is under $30 \%$ in the last $500 \mathrm{~m}$ and reaches $16 \%$ at the bottom. As time passes and gravitational compaction continues, the porosity at the deepest layer of the basin stays around $8 \%$ at $t=90 \mathrm{My}$ and $6 \%$ at $t=120 \mathrm{My}$.
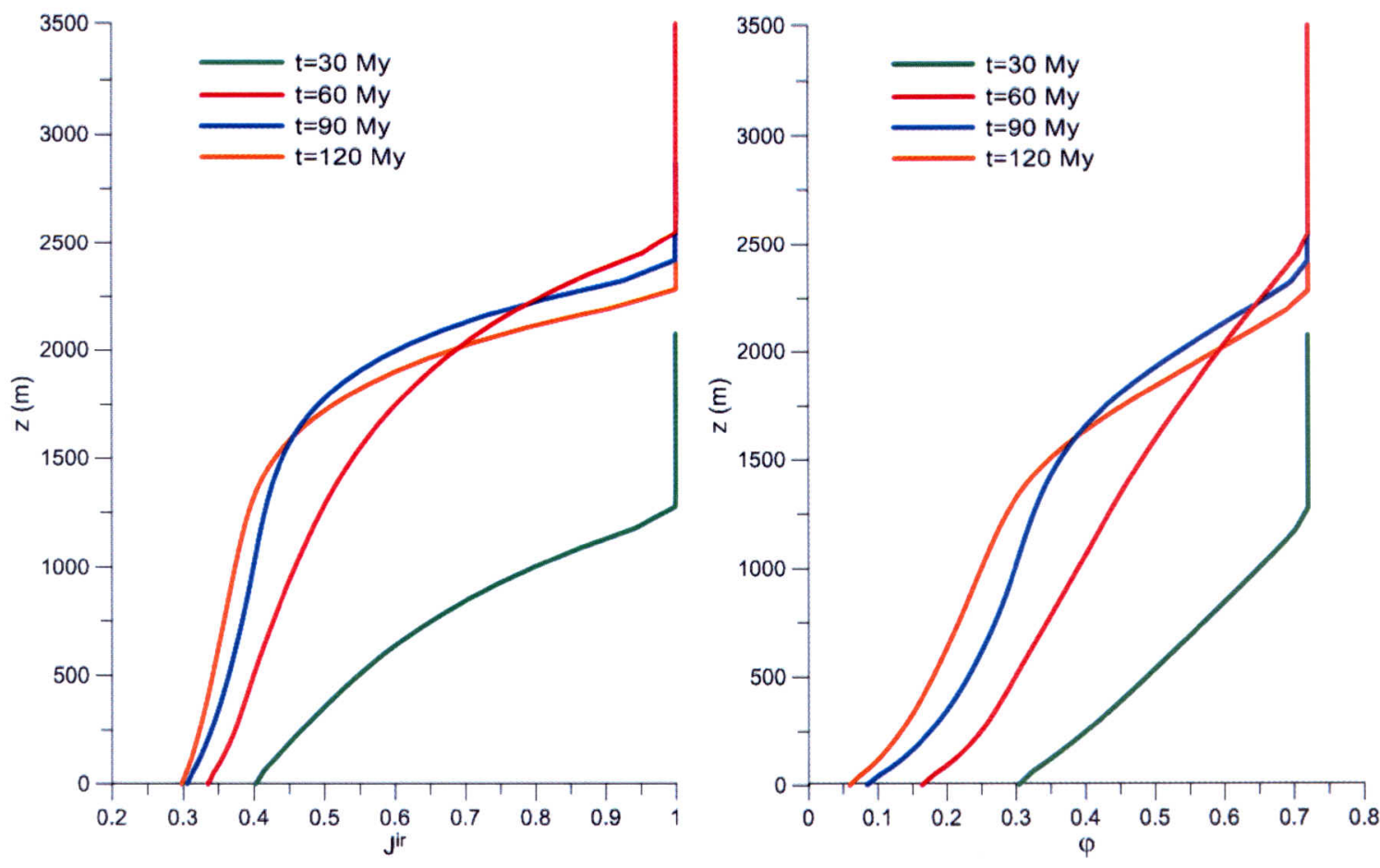

Figure 4. Jacobian of irreversible transformation profile (a); porosity profile (b)

Figure 5 presents the mean effective stress $p^{\prime}=(1 / 3) \operatorname{tr}\left(\underline{\underline{\sigma^{\prime}}}\right)$ and pore-pressure $p$ profiles. Comparing pore-pressure profile to hydrostatic values we observe that at the end of the accretion phase, $t=60 \mathrm{My}$, the basin presents excess pore-pressure through almost its entire height. As gravitational compaction evolves, the pore-pressure profile in the zones where purely mechanical deformation has occurred tend to return to the hydrostatic state, as shown in basin layers above $z=2000 \mathrm{~m}$ at $t=90 \mathrm{My}$.

Interestingly noting, from $t=90 \mathrm{My}$ to $t=120 \mathrm{My}$ the pore-pressure profile exhibits an increasing behavior in the deepest layers of the basin up to $z=300 \mathrm{~m}$. As described in previous works where only purely mechanical (i.e. plastic) deformation was considered (Maghous et al., 2013), a sedimentary basin submitted to gravitational compaction continuously dissipates the excess pore-pressure through time, transferring the load to the skeleton and reducing porosity. This behavior has changed in the deepest layers of the basin where chemo-mechanical (i.e. viscoplastic) deformation continued to decrease porosity but increased the excess pore-pressure as the effective stresses were relaxed. This means that after a certain moment, the velocity of excess pore-pressure dissipation has turned to be slower than the relaxation rate of effective stresses due to viscoplastic deformation. 

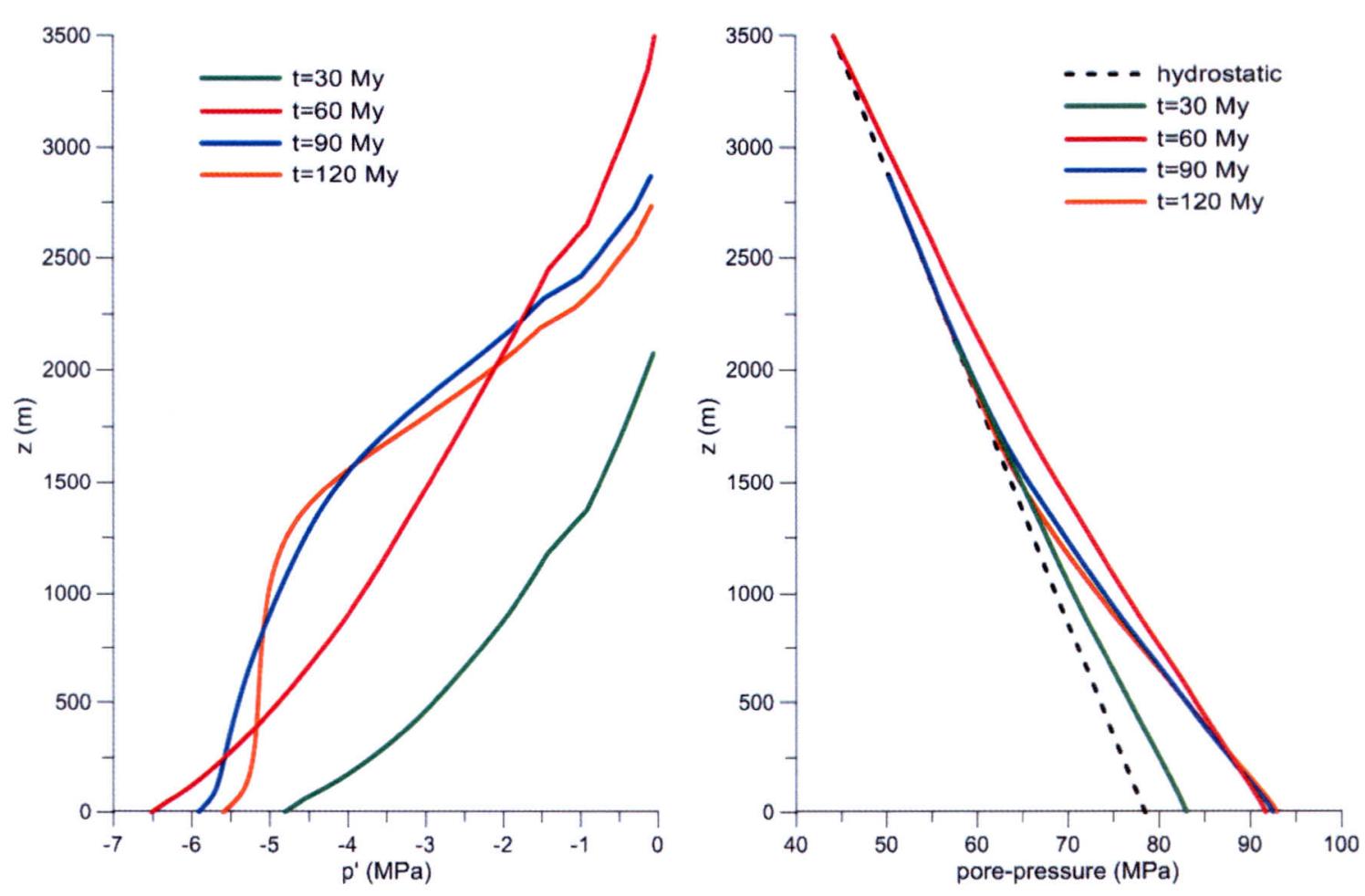

Figure 5. Mean effective stress profile (a); Pore-pressure profile (b)

Figures 6 exhibits the Jacobian of plastic and viscoplastic transformation, $J^{p}$ and $J^{v p}$ respectively. Concerning the evolution of the constitutive model, four different zones may be defined along the sedimentary basin depth associated to the deformation level of the sediment material (Maghous, 2009): zone A refers to sediment layers which remained in the elastic regime; zone $\mathrm{B}$ where purely mechanical (i.e. plastic) deformation has occurred; zone $\mathrm{C}$ denotes the transition zone where both mechanical and chemo-mechanical deformations occur; zone D where purely chemo-mechanical (i.e. viscoplastic) deformation is taking place after plastic deformation has been interrupted due to effective stress relaxation. The thickness of each zone for the different analyzed basin ages are presented in Table 1.

At $t=30 \mathrm{My}$ the upper elastic zone is $850 \mathrm{~m}$ thick, while the most part of the basin is comprised by zone B, where purely mechanical deformation occurs. There exists only a thin layer about $250 \mathrm{~m}$ where chemo-mechanical deformation occurs in conjunction with mechanical compaction. At $t=60 \mathrm{My}$ there is already a well-defined zone D which is $500 \mathrm{~m}$ thick at the bottom of the basin where solely chemo-mechanical deformation acts, followed by a transitional layer (zone C) defined between $z=500 \mathrm{~m}$ and $z=1250 \mathrm{~m}$ where both mechanical and chemo-mechanical deformations behave as interdependent processes. At this moment zone B is $1350 \mathrm{~m}$ thick and zone A is $900 \mathrm{~m}$ thick.

As time advances, both zones A, B and C decrease in thickness while zone D increases. At $t=120 \mathrm{My}$ the most part of the basin undergoes chemo-mechanical deformation induced by pressure-solution. This zone is defined from the bottom of the basin up to $z=1500 \mathrm{~m}$, followed by a $400 \mathrm{~m}$ thick transitional zone. The overall behavior of the sedimentary basin described herein are in good agreement with the description of several authors about purely mechanical and chemo-mechanical compaction phenomena and the interaction between them, as well as the corresponding thickness of each zone along the height of the basin. 

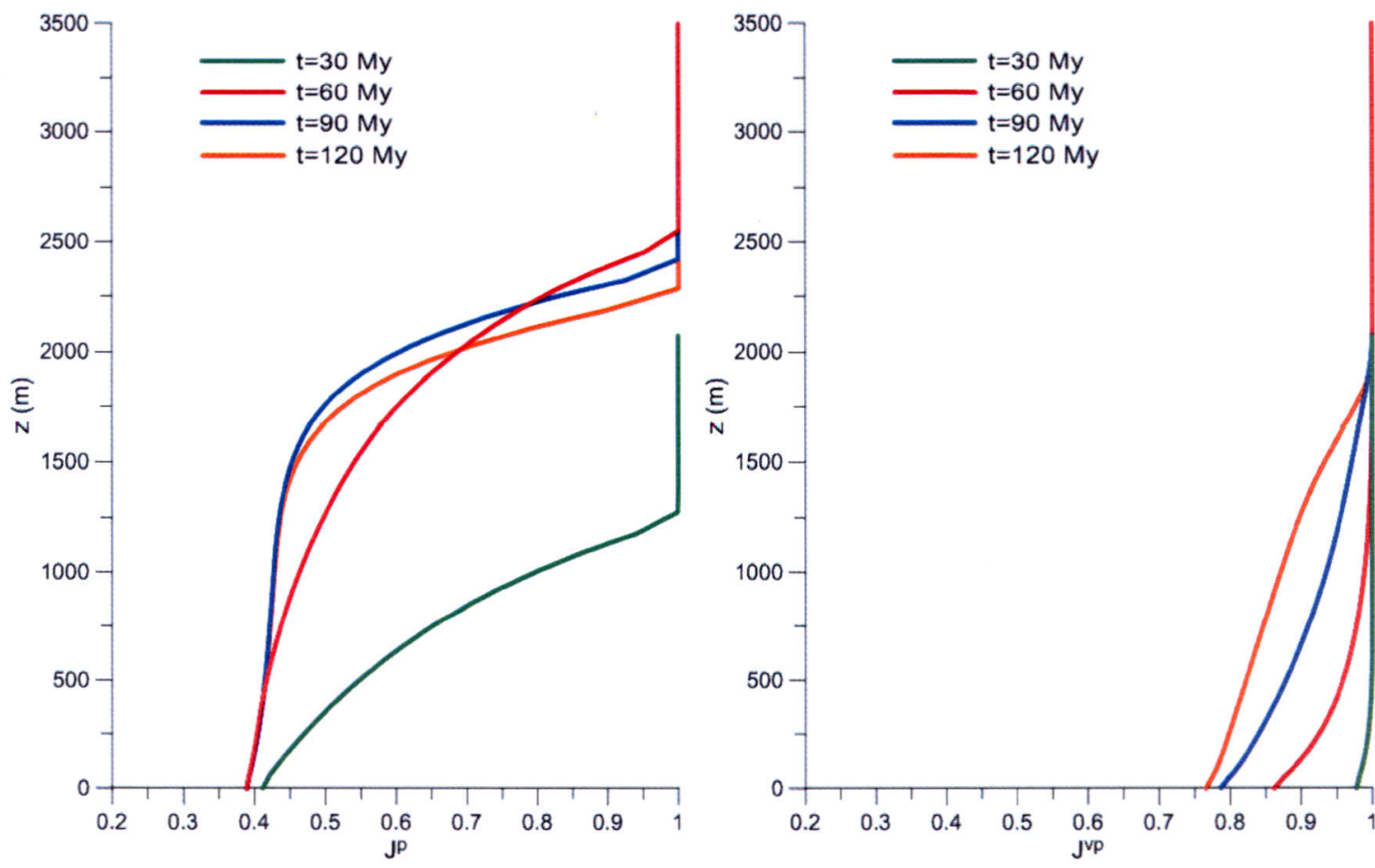

Figure 6. Jacobian of plastic transformation profile (a); Jacobian of viscoplastic transformation profile (b)

Table 1. Zones thickness for different basin ages

\begin{tabular}{|c|c|c|c|c|}
\hline & $\mathbf{t}=\mathbf{3 0} \mathbf{M y}$ & $\mathrm{t}=60 \mathrm{My}$ & $\mathrm{t}=90 \mathrm{My}$ & $\mathrm{t}=120 \mathrm{My}$ \\
\hline zone A & $\mathrm{z}=2100$ to $\mathrm{z}=1250 \mathrm{~m}$ & $z=3500$ to $z=2600 \mathrm{~m}$ & $\mathrm{z}=2850$ to $\mathrm{z}=2350 \mathrm{~m}$ & $\mathrm{z}=2730$ to $\mathrm{z}=2250 \mathrm{~m}$ \\
\hline zone B & $\mathrm{z}=1250$ to $\mathrm{z}=250 \mathrm{~m}$ & $\mathrm{z}=2600$ to $\mathrm{z}=1250 \mathrm{~m}$ & $\mathrm{z}=2350$ to $\mathrm{z}=2000 \mathrm{~m}$ & $\mathrm{z}=2250$ to $\mathrm{z}=1900 \mathrm{~m}$ \\
\hline zone $\mathrm{C}$ & $\mathrm{z}=250 \mathrm{~m}$ to $\mathrm{z}=0$ & $\mathrm{z}=1250$ to $\mathrm{z}=500 \mathrm{~m}$ & $\mathrm{z}=2000$ to $\mathrm{z}=1500 \mathrm{~m}$ & $\mathrm{z}=1900$ to $\mathrm{z}=1500 \mathrm{~m}$ \\
\hline zone D & - & $z=500 \mathrm{~m}$ to $z=0$ & $\mathrm{z}=1500 \mathrm{~m}$ to $\mathrm{z}=0$ & $\mathrm{z}=1500 \mathrm{~m}$ to $\mathrm{z}=0$ \\
\hline
\end{tabular}

\section{CONCLUSIONS}

Starting from the constitutive model formulated in (Bernaud et al., 2006) for the sediment material, chemo-mechanical deformation associated to Intergranular Pressure-Solution phenomenon was incorporated by means of additional viscoplastic terms in the state equations of the porous material, resulting in a poro-elasto-visco-plastic constitutive model with hydrochemo-mechanical coupling. The model is formulated within the framework of finite strains due to large porosity changes induced by the compaction process. Effects of large irreversible strains on the stiffness increase, on the hardening law as well as on the viscous law are accounted for through the reduction of the pore volume. The constitutive model is specifically devised for the sediment material subjected to mechanical and/or chemo-mechanical compaction. A 3D finite element numerical tool with a shared memory multiprocessing interface has been developed to simulate some fundamental processes on the formation history of an oceanic sedimentary basin such as the accretion phase, gravitational compaction and tectonic loading. 
Some issues remain to be addressed on both theoretical and computational viewpoints. At the current stage of this research, the influence of the geothermal gradient on the behavior of the sedimentary basin is the object of on-going research by addition of a thermo-mechanical coupling on the sediment constitutive model. The implementation of a finite element formulation with discontinuous interpolation functions is also a subject of interest to model fractures and faults in sedimentary basins, allowing the numerical tool to realize deeper investigations of lateral compressive and/or extensional movements caused by tectonics.

\section{REFERENCES}

Barthélémy, J. F.; Dormieux, L.; Maghous, S., 2003. Micromechanical approach to the modelling of compaction at large strains. Computers and Geotechnics, vol. 30, pp. 321-338.

Bathe, K. J., 1996. Finite element procedures. Prentice-Hall.

Bernaud, D.; Deudé, V.; Dormieux, L.; Maghous, S.; Schmitt, D. P., 2002. Evolution of elastic properties in finite poroplasticity and finite element analysis. International Journal for Numerical and Analytical Methods in Geomechanics, vol. 26, pp. 845-871.

Bernaud, D.; Dormieux, L.; Maghous, S., 2006. A constitutive and numerical model for mechanical compaction in sedimentary basins. Computers and Geotechnics, vol. 33, pp. 316329.

Coussy, O., 2004. Poromechanics. John Wiley \& Sons Ltd.

de Meer, S.; Spiers, C. J., 1997. Uniaxial compaction creep of wet gypsum aggregates. Journal of Geophysical Research, vol. 102, pp. 875-891.

Deudé, V.; Dormieux, L.; Maghous, S.; Barthélémy, J. F.; Bernaud, D., 2004. Compaction process in sedimentary basins: the role of stiffness increase and hardening induced by large plastic strains. International Journal for Numerical and Analytical Methods in Geomechanics, vol. 28, pp. 1279-1303.

Dormieux, L.; Maghous, S., 2000. Evolution of elastic properties in finite poroplasticity. C.R. Acad. Sci. Paris, vol. 328, n. Ilb, pp. 593-600.

Ghoussoub, J.; Leroy, Y. M., 2001. Solid-fluid phase transformation within grain boundaries during compaction by pressure solution. Journal of the Mechanics and Physics of Solids, vol. 49 , pp. $2385-2430$.

Gundersen, E.; Renard, F.; Dysthe, D. K.; Bjorlykke, K.; Jamtveit, B., 2002. Coupling between pressure solution creep and diffusive mass transport in porous rocks. Journal of Geophysical Research, vol. 107, pp. 19.1-19.19.

Hashin, Z., 1983. Analysis of composite materials - a survey. Journal of Applied Mechanics, vol. 50, pp. 481-505.

Hickman, S. H.; Evans, B., 1991. Experimental pressure solution in halite: the effect of grain/interphase boundary structure. Journal of the Geological Society, vol. 148, pp. 549-560. 
Hu, L. B.; Hueckel, T., 2007. Coupled chemo-mechanics of integranular contact: Toward a three-scale model. Computers and Geotechnics, vol. 34, pp. 306-327.

Maghous, S., 2009. A theoretical poro-elasto-visco-plastic model for mechanical and chemomechanical deformation in sedimentary basins. Solid Mechanics in Brazil: Rio de Janeiro, pp. 331-349.

Maghous, S.; Brüch, A.; Bernaud, D.; Dormieux, L.; Braun, A. L., 2013. Two-dimensional finite element analysis of gravitational and lateral-driven deformation in sedimentary basins. International Journal for Numerical and Analytical Methods in Geomechanics, vol. 38, pp. 725-746.

Perzyna, P., 1966. Fundamental problems in viscoplasticity. Advances in Applied Mechanics, vol. 9, pp. 243-277.

Rutter, E. H., 1976. The kinetics of rock deformation by pressure solution. Philosophical Transactions of the Royal Society of London, vol. 283, pp. 203-219.

Schmidt, V.; McDonald, D. A., 1979. The role of secondary porosity in the course of sandstone diagenesis. Aspects of Diagenesis, vol. 26, pp. 175-207.

Schneider, F.; Potdevin, J. L.; Wolf, S.; Faille, I., 1994. Modèle de compaction élastoplastique et viscoplastique pour simulateur de bassins sédimentaires. Revue de l'Institut Français du Pétrole, vol. 49, n. 2, pp. 141-148.

Schneider, F.; Potdevin, J. L.; Wolf, S.; Faille, I., 1996. Mechanical and chemical compaction model for sedimentary basin simulators. Tectonophysics, vol. 263, pp. 307-317.

Schutjens, P. M. T. M.; Spiers, C. J., 1999. Intergranular Pressure Solution in NaCl: Grain-toGrain Contact Experiments under the Optical Microscope. Oil \& Gas Science and Technology, vol. 54, pp. 729-750.

Tada, R.; Siever, R., 1989. Pressure solution during diagenesis. Annual Review of Earth and Planetary Sciences, vol. 17, pp. 89-118.

Weyl, P. K., 1959. Pressure Solution and the Force of Crystallization - A Phenomenological Theory. Journal of Geophysical Research, vol. 64, pp. 2001-2025.

Wood, D.M., 1990. Soil Behaviour and critical state soil mechanics. Cambridge University Press.

Yang, X. S., 2000. Pressure solution in sedimentary basins: effect of temperature gradient. Earth and Planetary Science Letters, vol. 176, pp. 233-243.

Zhang, X.; Spiers, C. J., 2005. Compaction of granular calcite by pressure solution at room temperature and effects of pore fluid chemistry. International Journal of Rock Mechanics \& Mining Sciences, vol. 42, pp. 950-960. 